\title{
Research Paper \\ The Effect of 12 Weeks Combined Training and Caffeine on Plasma Levels of Interleukin-1 $\beta$ and Interleukin 10 in Obese Men
}

\author{
Shirin Ghaderi Goodarzi ${ }^{1}$ (), ${ }^{*}$ Asieh Abbassi Daloii ${ }^{1}{ }^{~}$, Ahmad Abdi ${ }^{1}$ (), Ayoub Saeidi ${ }^{2}$
}

1. Department of Exercise Physiology, Faculty of Physical Education and Sport Sciences, Ayatollah Amoli Branch, Islamic Azad University, Amol, Iran. 2. Department of Exercise Physiology, Faculty of Physical Education and Sport Sciences, Damghan Branch, Islamic Azad University, Damghan, Iran.

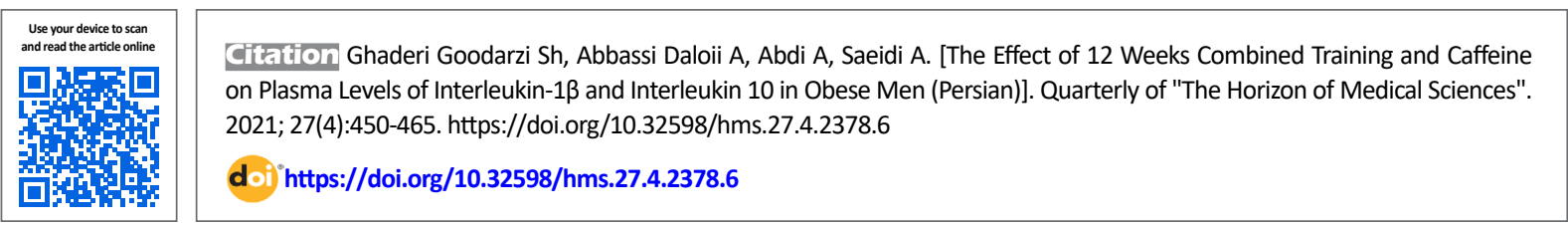

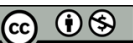

Received: 11 Dec 2020 Accepted: 27 Jan 2021 Available Online: 01 Oct 2021

Key words: Combined training, Caffeine, Interleukin$1 \beta$, Interleukin-10, Obesity

\section{ABSTRACT}

Aims Physical inactivity is associated with low-grade systemic inflammation and increased risk of chronic diseases. Beneficial effects of caffeine on adipocytes metabolism and its anti-inflammatory properties have been reported. This study aimed to evaluate the impact of 12 weeks of combined training and caffeine on plasma levels of inflammatory and anti-inflammatory indices in obese men.

Methods \& Materials In a quasi-experimental trial, 44 obese men were purposefully and accessibly selected and assigned into four groups each 11 samples: 1) control, 2) combined (resistance-endurance) training, 3) caffeine, and 4) combined training with caffeine. The study subjects received $6 \mathrm{mg}$ of caffeine per kilogram of body weight daily for 12 weeks. The combined training program was performed for 12 weeks, 3 sessions per week. The plasma levels of inflammatory (interleukin[IL]-1ß) and anti-inflammatory (IL-10) indices were measured using an ELISA kit. The obtained data were analyzed by dependent $t$ test, 1-way ANOVA, and Tukey post hoc test at the significant level of $P<0.05$.

Findings The results showed that 12 weeks of combined training, caffeine, and combined training-caffeine significantly reduced IL-1 $\beta$ levels and increased IL-10 in obese men at posttest compared to the pretest $(P=0.001)$. Also, changes in IL-1 $\beta$ and IL-10 levels were greater in the combined training-caffeine group $(P=0.001)$.

Conclusion Combined training with caffeine positively affects body composition and improves inflammatory and anti-inflammatory indices in obese people.

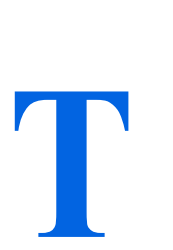

English Version

\section{Introduction}

he obesity epidemic is on the rise, and approximately one-third of the world's population is now overweight or obese [1]. Although genetic factors are involved in body weight, obesity is caused by many factors, including lifestyle, metabolism, and environmental factors. In obese people, excessive fat accumulation negatively affects almost all body's physiological functions. With the increase of fat cells, the production of inflammatory cytokines from fat cells increases. On the other hand, cytokines and anti-inflammatory chemokines decrease, leading to a mild systemic inflammation [2]. Interleukin(IL)-1 is an inflammatory cytokine with various physiological and pathological functions and plays an important role in health and disease. The levels of this interleukin in obese people are higher than people with a normal

* Corresponding Author:

Asieh Abbassi Daloii, PhD.

Address: Department of Exercise Physiology, Faculty of Physical Education and Sport Sciences, Ayatollah Amoli Branch, Islamic Azad University, Amol, Iran.

Tel: +98 (911) 1274366

E-mail: abbasi.daloii@gmail.com 
weight and are involved in developing chronic inflammation caused by obesity [5-3]. In contrast, IL-10 plays a key role in regulating the immune response and limiting inflammation. IL-10 suppresses inflammation through various mechanisms, including inhibiting the synthesis of inflammatory cytokines, such as IL-1 $\beta$, and suppressing nuclear factor (NF)- $\kappa \mathrm{B}$ and c-Rel activity in macrophages [6]. Evidence suggests that low circulating IL-10 is associated with obesity, cardiovascular disease, and type 2 diabetes [7]. In adults, IL-10 plays a protective role against obesity [8].

Exercise is recommended because it improves energy balance and potentially affects inflammatory signaling cascades. Regular exercise is associated with an anti-inflammatory status characterized by higher levels of antiinflammatory markers, such as IL-10, and lower levels of inflammatory cytokines, including IL-1 $\beta[9,10]$. According to studies, 8 weeks of resistance training causes a significant increase in IL-10 in obese women [10]. However, Fath Elahyan et al. did not observe a significant change in IL-10 levels in diabetic women after a period of combined (resistance-endurance) training despite a substantial decrease in IL-1 $\beta$ expression [11]. Also, in one study, the results showed that IL- $1 \beta$ did not change significantly after 8 weeks of resistance training in obese men [12].

In exercise, some herbal supplements boost muscle growth and burn fat. Caffeine (1,3,7-trimethylxanthine) is a heterocyclic organic compound with a purine base called xanthine, which consists of a pyrimidine ring attached to an imidazole ring [13]. It is an alkaloid because it is a plant metabolite derived from purine nucleotides and has a heterocyclic nitrogen atom [14]. Caffeine consumption stimulates consumers' consciousness, improves mood, and secrets catecholamines that benefit human behavior [15]. In addition, caffeine has other beneficial effects, such as antioxidant and anti-inflammatory properties that are very important for human health [16]. Caffeine consumption is associated with reduced inflammation in humans, and treatment of human macrophages with caffeine inhibits IL-1 $\beta$ secretion [17]. Caffeine has also been shown to increase serum levels of the anti-inflammatory cytokine IL-10 [18].

The effects of exercise on inflammatory factors have always been of interest to researchers. The anti-inflammatory effects of exercise can be caused by reducing fat accumulation. Metabolic pressure exerted by exercise correlates to the type, duration, and intensity of exercise. Since the sports activities vary in terms of intensity, duration, and organs involved, different exercise programs seem to affect weight loss and inflammation [19]. Besides all the positive effects of both types of endurance and resistance training, due to more variety and less pressure, combined (resistance-endur- ance) exercise has a greater impact on weight loss and body fat mass than each of these exercises alone [20]. On the other hand, caffeine can be effective in reducing inflammation and improving the body composition of obese people by reducing inflammatory factors. However, the interactive effects of combined exercise and caffeine on inflammatory and anti-inflammatory agents in obese individuals are unclear. Therefore, considering the potential benefits of caffeine and regular physical exercise in reducing obesity and the potentially beneficial consequences of their interactive effect, this study investigated the impact of 12 weeks of combined exercise and caffeine on plasma inflammatory and anti-inflammatory parameters in obese men.

\section{Materials and Methods}

The present research is an applied study, and its method is quasi-experimental. This study was approved by the Ethics Committee with the number IR.IAU.M.REC.1399.009 in Islamic Azad University, Ayatollah Amoli Branch in 2020. The study population consists of obese male volunteers in Tehran selected through a call in public and administrative centers. Accordingly, after initial clinical evaluations, including taking history of cardiovascular disease, clinical and diagnostic examinations, 44 obese men (aged 23-32 years) with a body mass index above 30 who were not on drug treatment were selected based on the inclusion criteria. The study subjects completed a health form to ensure disease-free and personal satisfaction. The inclusion criteria were no chronic diseases according to the medical history questionnaire (cardiovascular disease, diabetes, various cancers, and kidney and gastrointestinal disorders or any injury or problem that restricts them from engaging in physical activity), with $\mathrm{BMI} \geq 30$, and waist-to-height ratio $>0.5$ and no history of exercise in the last six months, no record of sleep disorders, no smoking and not taking any supplements, alcohol, caffeinated substances, and drug treatment. The exclusion criteria were the absence of more than one session in the exercise program, the occurrence of accidents, injuries, contracting diseases, and the occurrence of any interfering factor that affected the effective participation of the subjects in the training sessions. In a separate session after the medical examination, the purpose of the study and how to perform it were explained to the subjects. After filling in the personal information questionnaire and signing the consent form, each subject came to the test site the next day to perform the tests. At the beginning of the session, anthropometric features, including height, weight, and body composition, were measured. In the second session, the subjects were tested for determining a maximum repetition. Subjects were then homogeneously divided into 4 groups based on maximum repetition and demographic 
characteristics (each group with 11 people): 1) control, 2) caffeine, 3) exercise (resistance-endurance), and 4) caffeine plus exercise. After three days, the subjects went to the laboratory, and their blood samples were taken to assess the levels of IL-1 $\beta$ and IL-10. The experimental groups then performed a 12-week combined exercise and caffeine intervention program. In the end, anthropometric and blood sampling features were obtained again.

\section{Exercise protocol}

The combined (resistance-endurance) exercise protocol was performed for 12 weeks, 3 sessions per week. Subjects in the training group, after 5 minutes of warm-up, performed circular resistance activity (3 sets with 14 repetitions in $50 \%$ of one maximum repetition and 30 seconds rest between each station and 2 minutes rest between each turn for 6 stations) (1-3). Stations included squats, chest presses, back of the machine legs, shoulders, ankles, and boat [21]. Then, after 10 minutes of rest, they performed aerobic activity with an intensity of $70 \%$ of the maximum heart rate for 30 minutes. To determine a maximum of one repetition (1RM), the subjects of the 1RM resistance training group were taken by the Barziski method. In this way, weight is selected for the subject that the subjects do a maximum of 6-8 repetitions. The weight was then placed in the appropriate formula with the repetitions. Measurements were taken for all 6 stations.

\section{Caffeine consumption}

The caffeine group received $6 \mathrm{mg}$ of caffeine per kilogram of body weight per day (manufactured by the German company Merck and licensed by the Ministry of Health with registration number 0225.02584 .01 ). Subjects in the caffeine with exercise group consumed $3 \mathrm{mg}$ per $\mathrm{kg}$ body weight twice a day on training days, 90 minutes before and 30 minutes after the exercise. The control group also continued their daily activities and only received a placebo of 6 mg per kilogram of body weight [22].

\section{Sampling and measurement of laboratory variables}

To evaluate biochemical variables, blood sampling was performed after 12 to 14 hours of fasting before and 12 weeks after the intervention (72 hours after the last training session). At each stage, $10 \mathrm{~mL}$ of blood was taken from the brachial vein of the subjects' right arm at rest and in a sitting position by a laboratory expert. Blood samples were stored at $-80^{\circ} \mathrm{C}$ after centrifugation, and plasma separation was done after the tests were performed. To prevent the effect of circadian rhythm, blood sampling was performed at certain times of the day ( 8 AM to $10 \mathrm{AM}$ ). Plasma IL-
$1 \beta$ was measured using an ELISA kit (Abcam Company of America) with a detection range of $15.6-500 \mathrm{pg} / \mathrm{mL}$, a sensitivity of $6.5 \mathrm{pg} / \mathrm{mL}$, and a coefficient of variations of 4.5\%-8.7\%. Plasma IL-10 was measured using an ELISA kit (Abcam Company of America) with a detection range of $9.4-3000 \mathrm{pg} / \mathrm{mL}$, the sensitivity of $1.4 \mathrm{pg} / \mathrm{mL}$, and coefficient of variations of $3.2 \%-5.2 \%$

\section{Statistical analysis}

The Shapiro-Wilk test was used to ensure the normal distribution of variables. After the normality of the data distribution was determined, the dependent t test, 1-way analysis of variance, and Tukey's post hoc test were used to compare the mean changes in protein levels of the groups. The significance level was considered $\mathrm{P} \leq 0.05$ in all cases. All statistical operations were performed with SPSS software version 23 .

\section{Results}

Table 1 indicates the mean and standard deviation of anthropometric characteristics of subjects in different groups. Analysis of the results of plasma IL-1 $\beta$ in obese men using repetitive analysis of variance with intergroup factor showed that regardless of the type of group, there was a significant difference between before and after 12 weeks of the research period in plasma IL-1 $\beta$ value $(\mathrm{P}<0.001)$. Also, the interaction between the studied groups and the time of the study showed a significant difference in plasma IL-1 $\beta$ levels $(\mathrm{P}<0.0001)$. The highest change in plasma IL-1 $\beta$ levels was observed in the combined training with caffeine group and the lowest in the caffeine group. Based on the results of the Tukey post hoc test, plasma IL-1 $\beta$ levels had a significant decrease in the combined training group, caffeine group, and combined training-caffeine compared to the control group $(\mathrm{P}=0.001)$. Plasma IL-1 $\beta$ levels were significantly reduced in the combined training with caffeine group compared to the caffeine and combined training groups $(\mathrm{P}=0.0001)$. Also, plasma IL- $1 \beta$ levels significantly decreased in the combined training group compared to the caffeine group $(\mathrm{P}=0.003)$. The results of the paired t test to examine the intragroup changes in plasma IL-1 $\beta$ levels showed that the difference between before and after in the combined training, caffeine, and combined with caffeine training groups were significant $(\mathrm{P}<0.0001, \mathrm{P}<0.0001$, $\mathrm{P}<0.0001$, respectively) (Figure 1).

Analysis of the results obtained on plasma IL-10 levels in obese men using repeated-measures ANOVA with intergroup factor showed that regardless of the type of study, there is a significant difference between times before and after 12 weeks of the study period in plasma IL-10 levels (P 


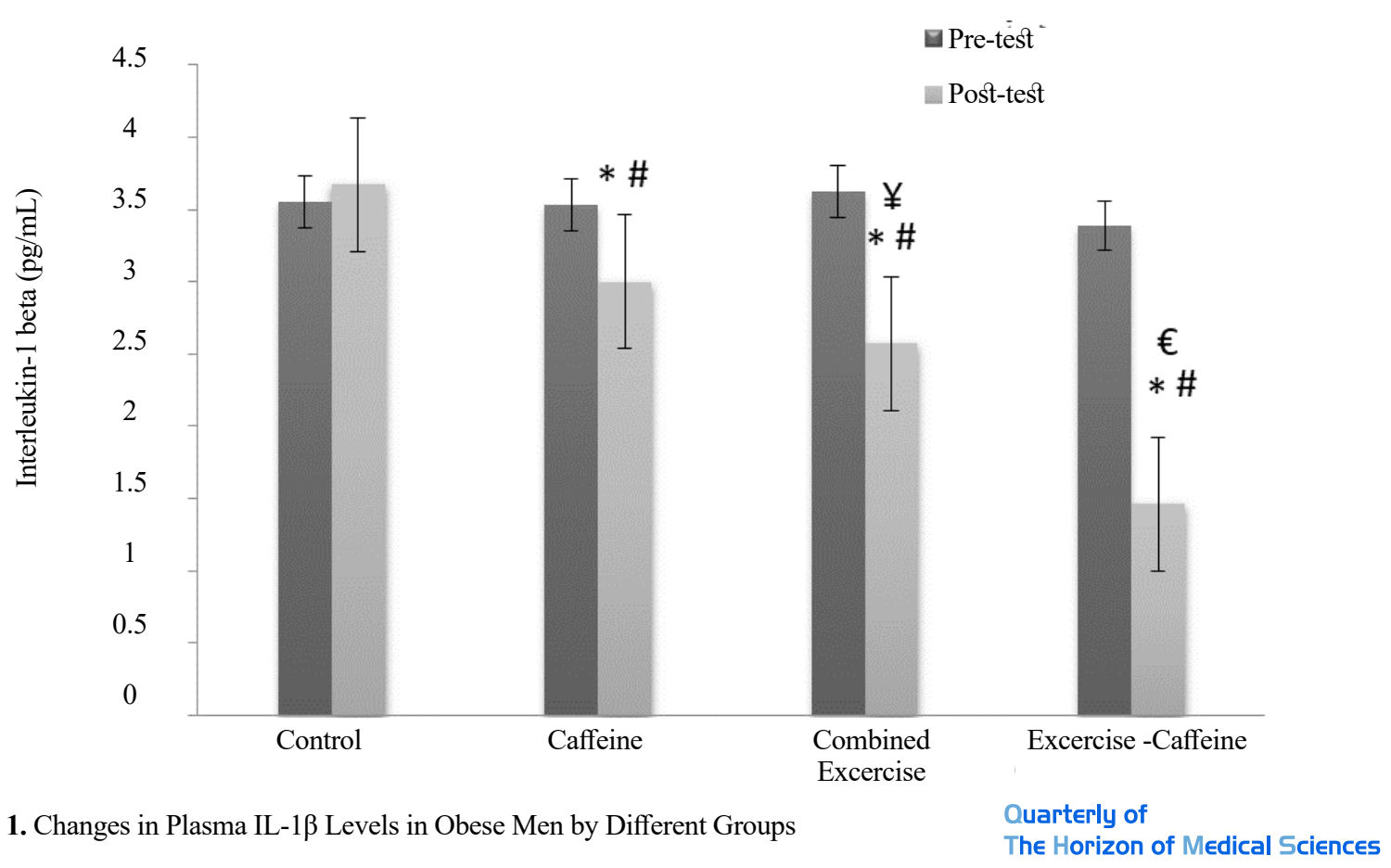

Figure 1. Changes in Plasma IL-1 $\beta$ Levels in Obese Men by Different Groups

Quarterly of

The Horizon of Medical Sciences

*Significant difference compared to pretest, \# significant difference compared to the control group, ¥ Significant difference compared to the caffeine group, $€$ Significant difference compared to the caffeine group and combined exercise $(\mathrm{P} \geq 0.05)$.

$<0.001)$. Also, the interaction between the studied groups and the time of the study showed a significant difference in plasma IL-10 levels $(\mathrm{P}<0.0001)$. The highest change in plasma IL-10 levels was observed in the combined training with caffeine group, and the lowest was observed in the caffeine group. Based on the results of the Tukey post hoc test, plasma IL-10 levels had a significant increase in the combined training group, caffeine, and combined training with

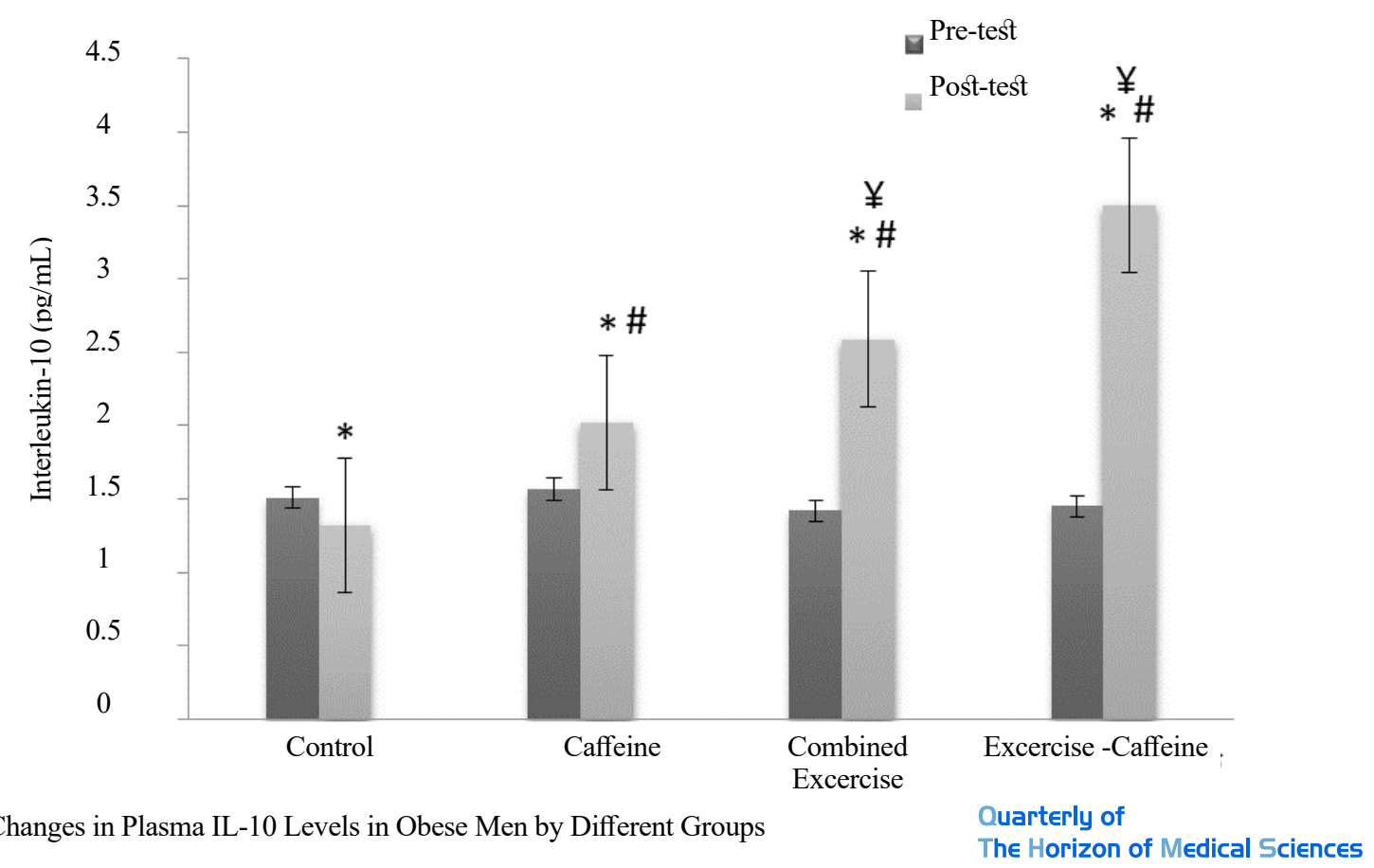

Figure 2. Changes in Plasma IL-10 Levels in Obese Men by Different Groups

* Significant difference compared to the pretest, \# Significant difference compared to the control group, $¥$ Significant difference compared to the caffeine group $(\mathrm{P} \geq 0.05)$. 
Table 1. The Mean and Standard Deviation of anthropometric characteristics of subjects in different groups

\begin{tabular}{|c|c|c|c|c|}
\hline \multirow{2}{*}{ Group } & \multicolumn{4}{|c|}{ Mean \pm SD } \\
\hline & Control & Caffeine & Combined Training & Combined Training - Caffeine \\
\hline Age (y) & $27 \pm 3$ & $28 \pm 2$ & $27 \pm 3$ & $29 \pm 4$ \\
\hline Height $(\mathrm{cm})$ & $170.22 \pm 5.61$ & $169.87 \pm 4.91$ & $174.23 \pm 4.6$ & $171.66 \pm 9.00$ \\
\hline Weight (kg) & $98.91 \pm 8.40$ & $96.10 \pm 9.51$ & $97.36 \pm 10.21$ & $95.42 \pm 9.46$ \\
\hline Body mass index $\left(\mathrm{kg} / \mathrm{m}^{2}\right)$ & $33.91 \pm 3.54$ & $33.68 \pm 2.48$ & $32.33 \pm 3.33$ & $32.53 \pm 4.74$ \\
\hline
\end{tabular}

caffeine compared to the control group $(\mathrm{P}=0.001)$. Also, plasma IL-10 levels increased significantly in the combined training and combined training with caffeine groups compared to the caffeine group $(\mathrm{P}=0.001)$. The results of the paired $t$ test to examine the intragroup changes in plasma IL-10 levels showed that the difference between before and after in the control groups $(\mathrm{P}=0.014)$, combined training, caffeine, and combined training with caffeine is significant ( $\mathrm{P}<0.001, \mathrm{P}<0.001, \mathrm{P}<0.001$, respectively ) (Figure 2 ).

\section{Discussion}

The current study results showed that 12 weeks of combined exercise intervention significantly reduced IL-1 $\beta$ levels and increased IL-10 in obese men. Significant decrease in IL-1 $\beta$ levels and increase in IL-10 in obese men in the current study are consistent with the results of some previous studies $[9,10,23,24]$. The results of Balducci et al. show that IL-1 $\beta$ decreases when people perform high-intensity aerobic and resistance training for 12 months. However, anti-inflammatory cytokine (IL-10) was increased only in the combined training group [23]. These results suggest that the anti-inflammatory effects of exercise are related to the nature of the exercise and its duration. Combined training (aerobic and resistance) is much more effective in reducing inflammatory markers than resistance or aerobic training alone, despite consuming the same calories. The exact mechanism of the effects of training on reducing inflammation is not yet fully understood. However, training protocols that reduce body fat and significantly improve body mass index are effective in reducing inflammatory factors and increasing anti-inflammatory factors [25]. IL$1 \beta$ is an inflammatory cytokine that is chronically elevated in obesity and can destroy $\beta$ cells and alter insulin sensitivity [26]. IL-1 $\beta$ is positively correlated with body mass index (BMI) [27], so after 12 weeks of combined training, weight and body mass index decreased, which could be a possible reason for the results. IL-10 is more elevated in response to an increase in inflammatory cytokines to suppress them. One of the reasons for the increase in IL-10 after training is the increase in fat oxidation and thus the reduction of adipose tissue, including splanchnic fat. It has been shown that a decrease in adipose mass along with a reduction in the penetration of macrophages into adipose tissue and the conversion of M1 macrophage monocytes to the M2 macrophage monocyte phenotype causes antiinflammatory cytokines, such as IL-10 to increase and proinflammatory cytokines to decrease [28]. Another mechanism involved in increasing IL-10 is an increase in IL-6 due to training. Training increases muscle metabolism and leads to an increase in IL- 6 in muscle and blood. A high IL-6 level increases IL-10 secretion in macrophages [29]. In addition, long-term exercise reduces the production of atherogenic cytokine mononuclear cells, such as tumor necrosis factor-alpha (TNF- $\alpha$ ) and interferon-gamma (IFN- $\gamma$ ), while increases producing anti-inflammatory cytokines, such as IL-10 [30]. These multiple effects of exercise turn resting cytokine balances into anti-inflammatory states. According to molecular mechanisms, by negatively regulating the activity of nuclear factor-kappa bi (NF- $\mathrm{kB})$, the exercise increases the secretion of IL-10 by monocytes and $\mathrm{T}$ cells through the Th2 pathway [31]. Contrary to the results of the current study, no significant change in IL-1 $\beta$ was reported after 10 weeks of resistance and aerobic training in inactive and overweight young men [32]. The inconsistency can be attributed to the baseline level of cytokines and the type of training. A base level of cytokines and repetition of training affect the results. In another study by Rahimian et al., after 8 weeks of resistance training with an intensity of $60 \%$ to $85 \%$ of a maximum repetition, a significant change was not observed in IL-10 levels in obese women [33]. The discrepancy in the reported research can be related to factors, such as the health status and initial weight of the subjects and the insufficient duration of the training period. It seems that resistance training in the current study can be a good way to reduce chronic inflammation in obese people. 
The current study results also showed that 12 weeks of caffeine consumption significantly reduced IL-1 $\beta$ levels and increased IL-10 in obese men. Few studies have shown that chronic caffeine consumption reduces inflammatory damage and chronic inflammation in the liver and brain $[34,35]$, consistent with the current study results. These studies have attributed the protective effect of caffeine to its antioxidant properties and reduced activation of resident macrophages (Cooper cells) and microglia. In addition, caffeine consumption reduces the expression of inflammatory cytokines in blood monocytes and macrophages and suggests that it may locally reduce chronic inflammation (expression of inflammatory cytokine IL-1 $\beta$ ) [36-38]. Studies have shown that caffeine, as an oral alkaloid in coffee, blocks adenosine receptors, increases cyclic adenosine monophosphate, decreases the production of free bases, produces prostaglandins, and expresses the gene for inflammatory factors and the production of proinflammatory cytokines. The current study results also support that caffeine reduces chronic inflammation in obese people. In the current study, the combined training for 12 weeks with caffeine had more beneficial effects on body composition and improved plasma interleukin-1 beta (IL-1 $\beta$ ) and interleukin-10 (IL-10) levels in obese men. These findings are consistent with the results of some previous research $[18,39]$. The effect of regular caffeine consumption, physical activity, and inactivity (including any sedentary activity) on the inflammatory status in healthy people has been studied. Caffeine consumption was a negative predictor of anti-inflammatory protein [18]. One of the possible mechanisms involved in changing the level of IL-10 by caffeine is increasing the level of cyclic adenosine monophosphate in muscle [40]. Caffeine is an adenosine receptor antagonist. Chronic caffeine consumption may increase the expression of adenosine A2A receptors and extracellular adenosine levels. Therefore, increasing ADA activity indicates compensatory properties to reduce adenosine levels and neutralize caffeine antagonist function [41]. Caffeine consumption during training can increase changes in the immune response and facilitate lipolysis and glycogenolysis to provide energy to skeletal muscle and other tissues during and after the exercise. Caffeine may improve training performance by inhibiting glycogen phosphorylase enzymes and reducing glycogen deficiency [42]. In addition, caffeine accelerates the release of $\beta$-endorphins, epinephrine, cortisol and increases IL-6 levels, and these changes in the immune response contribute to the benefits of caffeine in training performance [40]. Caffeine supplementation with combined training has been more effective in reducing the inflammatory status of obese subjects than either of these interventions alone. Therefore, caffeine consumption in combination with training is likely to have more benefits for obese people prone to inflammation. Combined training was one of the strengths of the current study because it can have different responses and adaptations than other training programs. There were some limitations in the current study, such as the lack of measurement of other inflammatory and anti-inflammatory factors in obese subjects. However, more research is needed in this area.

\section{Conclusion}

Briefly, the current study results showed that combined training with caffeine has a beneficial effect on body composition and improves inflammatory and anti-inflammatory indicators in obese people. It is suggested that combined training and caffeine intervention be considered a treatment method to reduce chronic inflammation in obesity.

\section{Ethical Considerations}

\section{Compliance with ethical guidelines}

This research was carried out with the approval of the Ethical Committee of the Islamic Azad University, Ayatollah Amoli Branch (IR.IAU.M.REC.1399.009).

\section{Funding}

This research is extracted from a $\mathrm{PhD}$ dissertation that has been conducted with the support of the Vice Chancellor for Research of the Islamic Azad University, Ayatollah Amoli Branch.

\section{Authors' contributions}

All authors contributed to the current article, equally.

\section{Conflicts of interest}

The authors declared no conflict of interest. 
This Page Intentionally Left Blank 


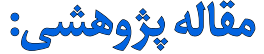

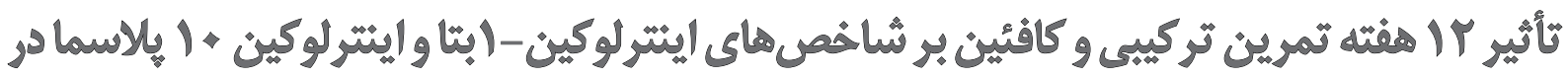

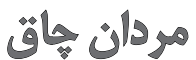

شيرين قادرى كودرزى' هـ، "آسيه عباسى دلويى' ــ، احمد عبدى' ــ ايوب سعيدى'

ا. كروه فيزيولورثى ورزشى، دانشكده تربيتبدنى و علوم ورزشى، واحد آيتالهه آملى، دانشكاه آزاد اسلامى، آمل، ايران.

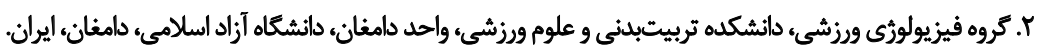

\section{حكSי}

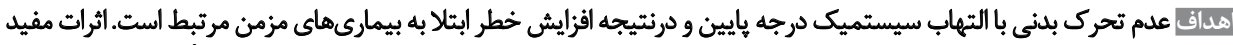

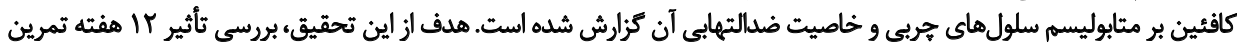

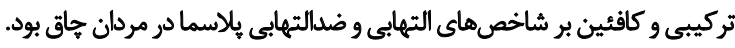

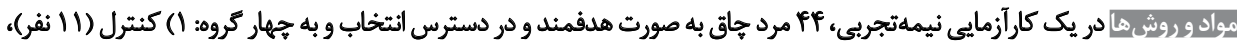

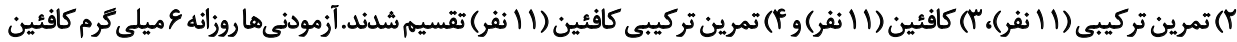

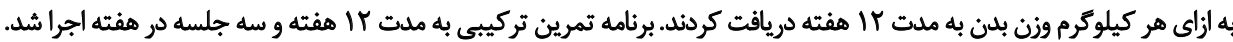

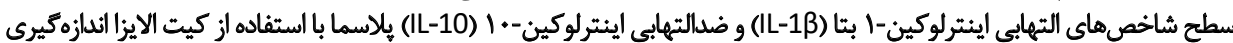

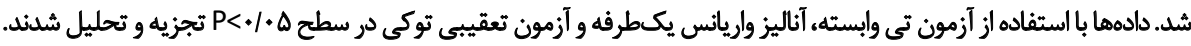

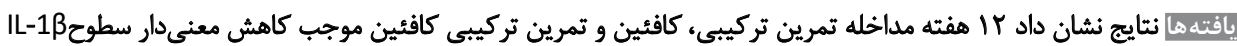

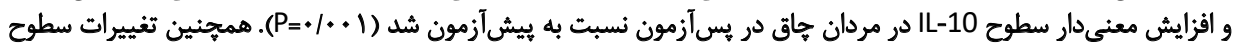

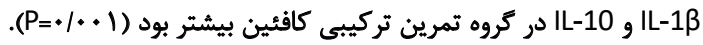

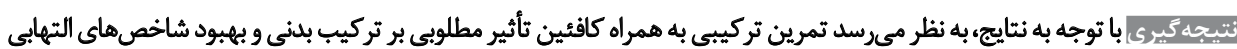
و وضدالتهابي در افراد جاقي دارد.

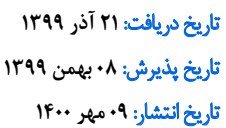

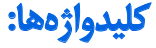
تمرين تركيبي، كافنين،

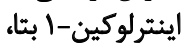
اينترلوكين-• ا، جإقى

است و نقش مهمى در سلامتى و بيمارى دارد. مقادير اين

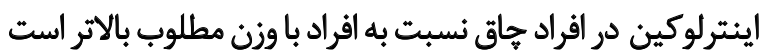

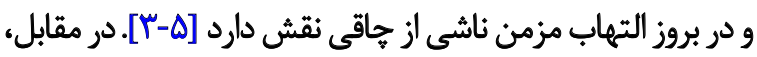

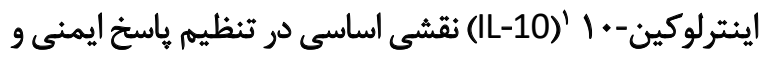

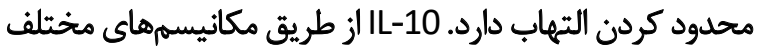

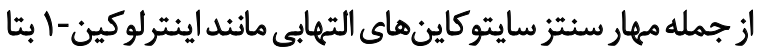

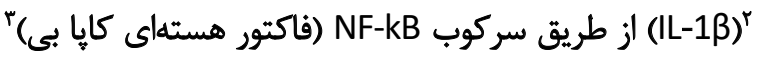

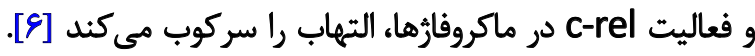

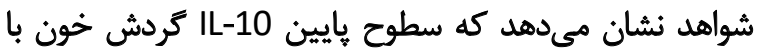

1. Interleukin-10

2. Interleukin-1 $\beta$

3. Nuclear Factor-kB (NF-kB)

مقدمه

إيدمى جاقي در حال افزايش است و تقريباً يكسوم جمعيت

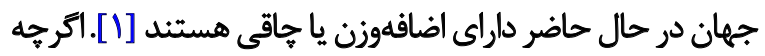

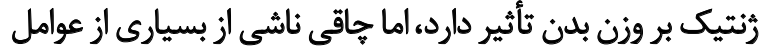

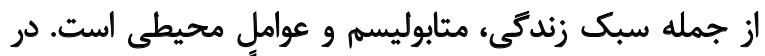

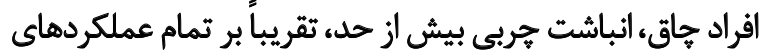

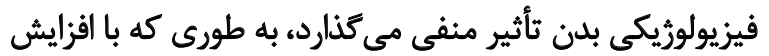

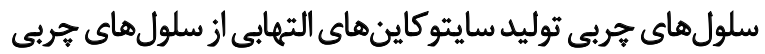

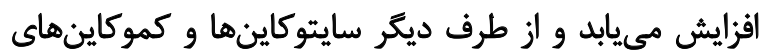

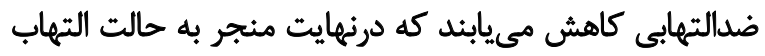

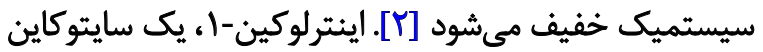

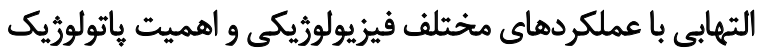


افراد جاق مؤثر باشد. با اين حال، اثرات تعاملى تمرين تركيبى

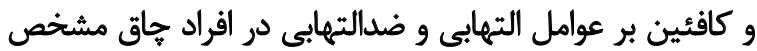

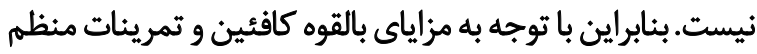

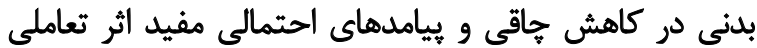

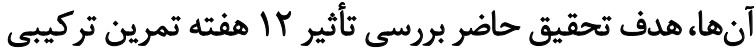

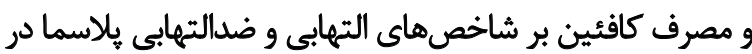

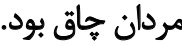

مواد و روشئ.

يثروهش حاضر از نوع كاربردى و روش آن نيمهتجربى است.اين

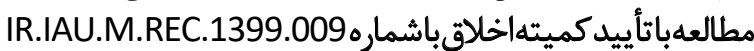

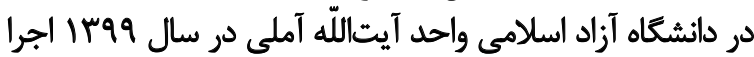

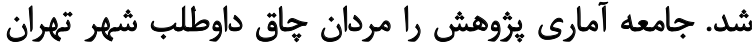

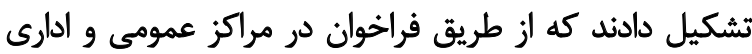

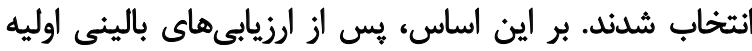

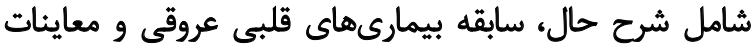

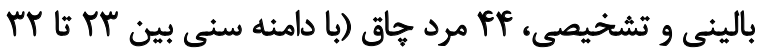

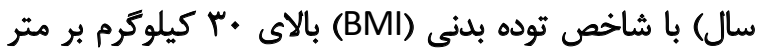

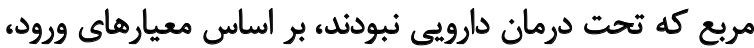

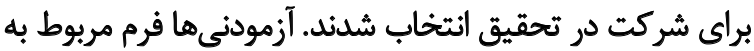

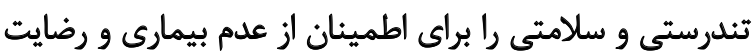

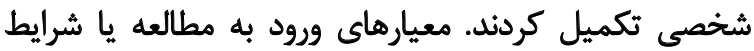

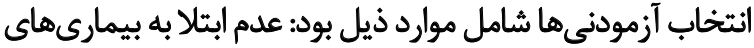

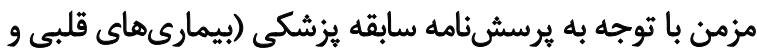

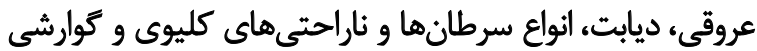

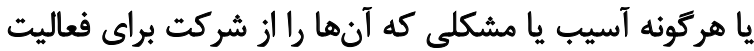

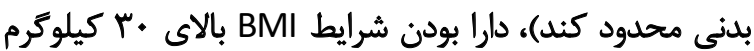

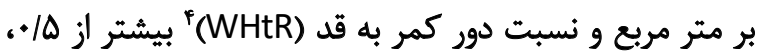

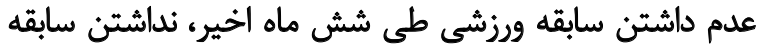

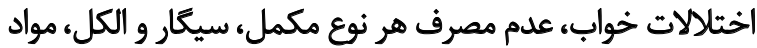

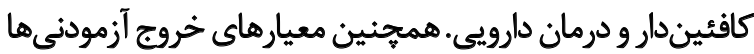

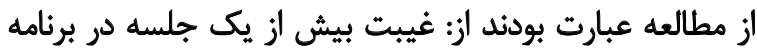

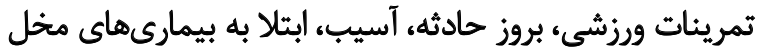

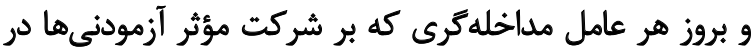
جلسات تمرين اثركذار باشد.

در يك جلسه جداكانه بعد از انجام معاينات يزشكى، هدف از از إز

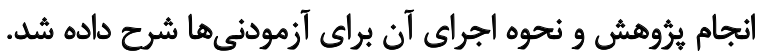

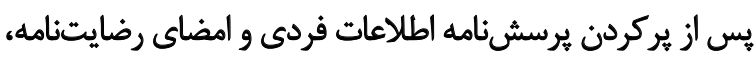

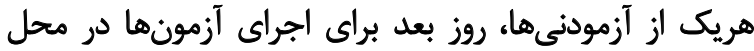

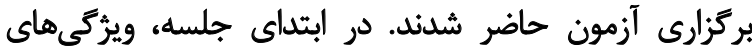

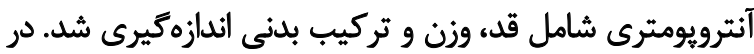

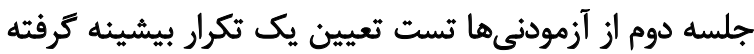

4. Waist-to-Height Ratio (WHtR)

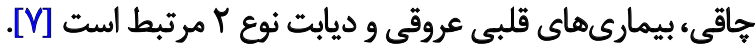

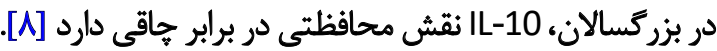
تمرينات ورزشى به دليل بهبود تعادل انرئى و تأثيرات

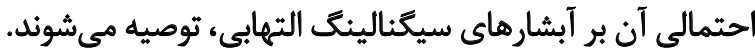

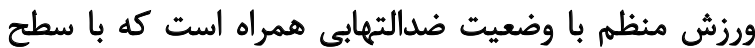

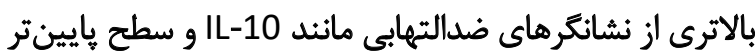

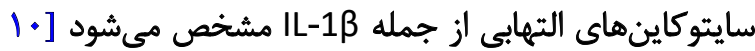

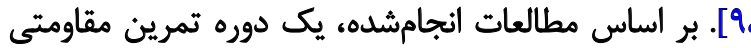

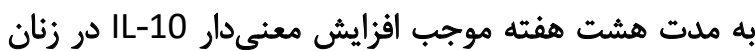

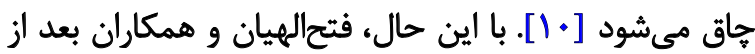
يك وهله تمرين تركيبى (مقاومتى استقامتى) على مغرغم كاهش

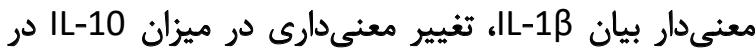

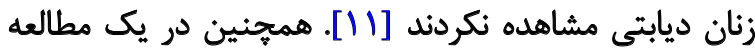

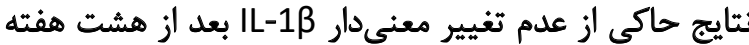

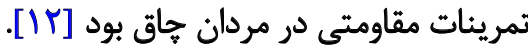

در ورزش، برخى مكملهاى تياهى براى تقويت رشد عضلات

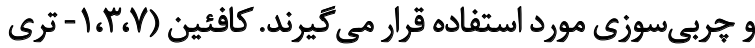

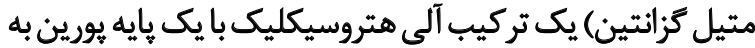

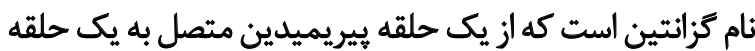

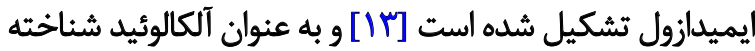

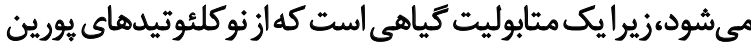

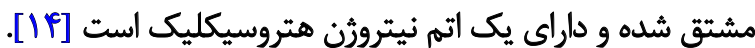

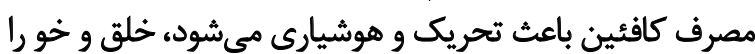

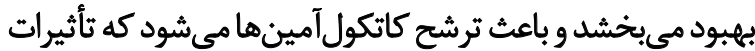

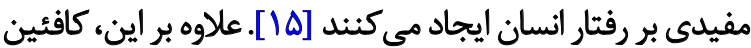

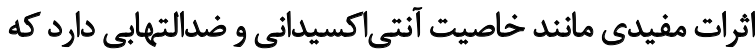

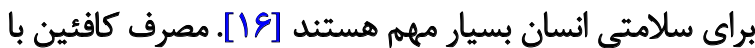

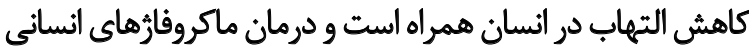

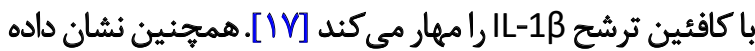

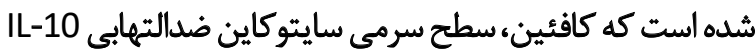

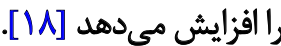

بررسى اثرات ورزش بر عوامل التهابى همواره مورد توجه

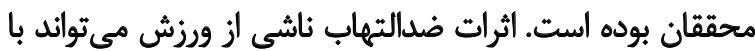

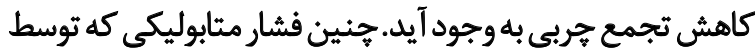

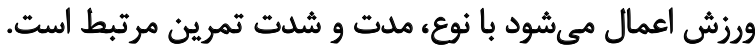

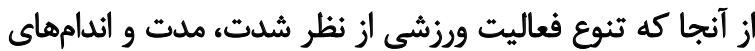

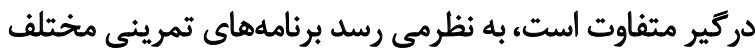

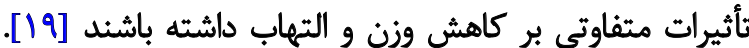

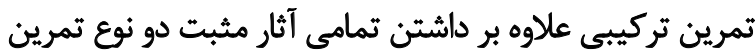

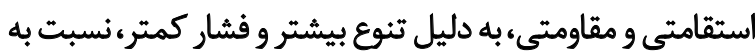

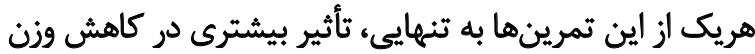

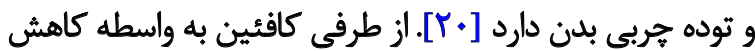

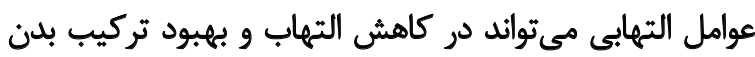




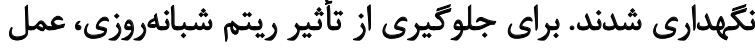

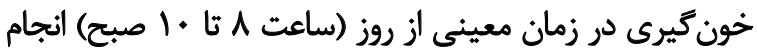

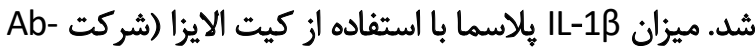

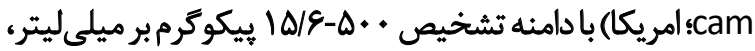

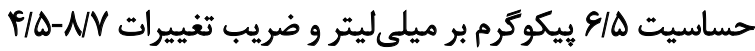

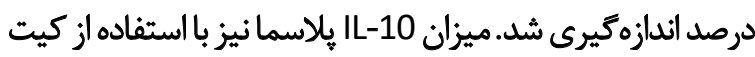

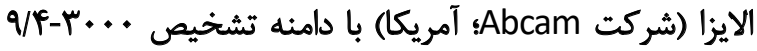

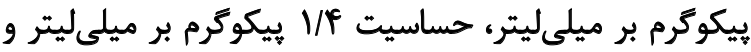

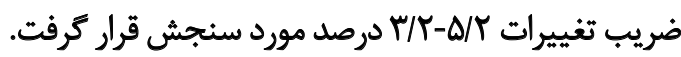

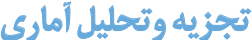

براي اطمينان از طبيعى بودن توزيع متغيرها، از آزمون شاييرو

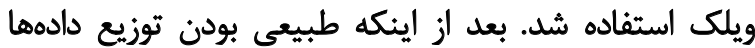

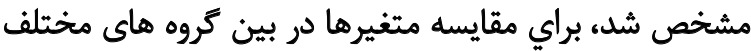

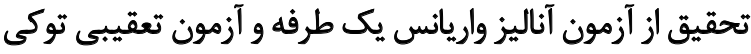

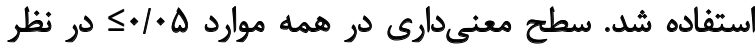

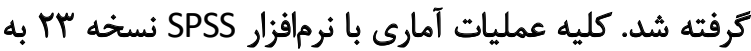

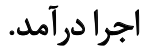

يافتهمها

در جدول شماره 1 ميانكين و انحرافمعيار ويرُكىهاي آنترويومتريك آزمودنى هادر كروههاي مختلف نشان دانين اده شده است.

نتايج آزمون تحليل واريانس يكسرفه نشان ميدهد بين بين

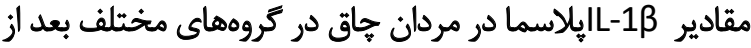

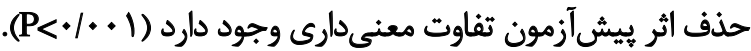

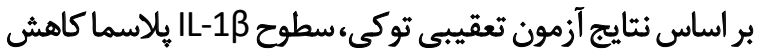

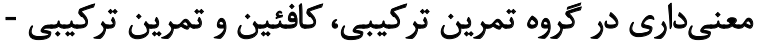

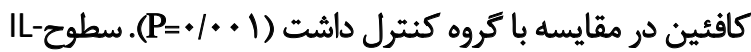

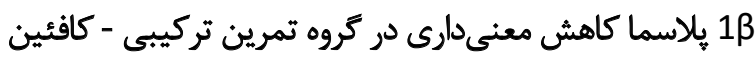

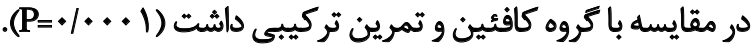

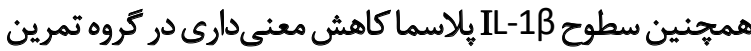

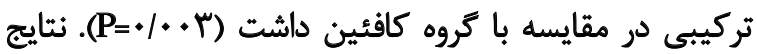

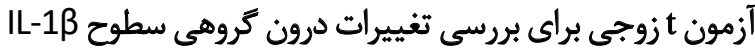

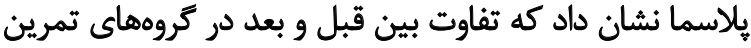

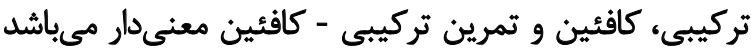

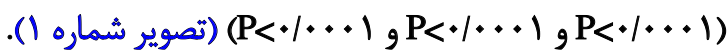

همجنين نتايج آزمون تحليل واريانس يكطرفه نشان مى دهد

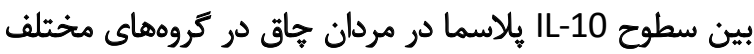

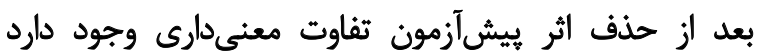

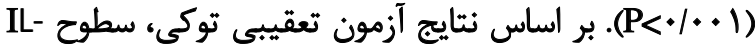

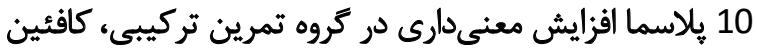

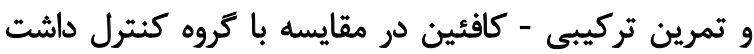

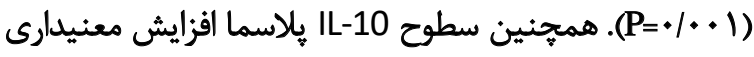

شد. سيس آزمودنىها به صورت همكَن بر اساس حداكثر تكرار

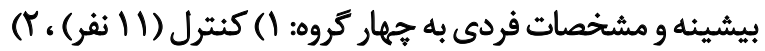

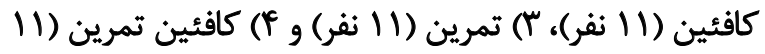

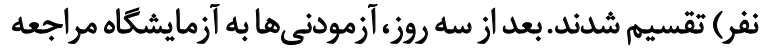

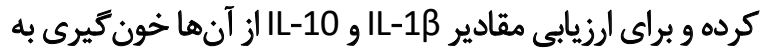

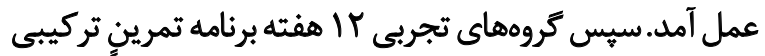

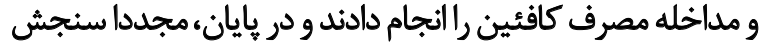
ويزّكى هاى آنترويومترى وخون خيرى انجام شد.

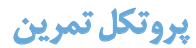

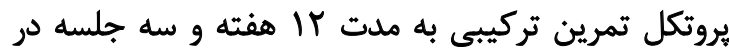

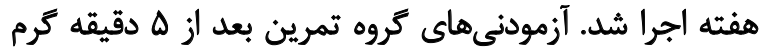

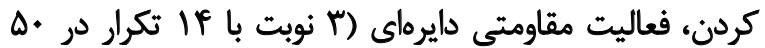

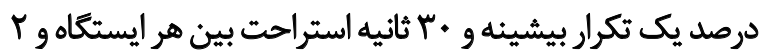

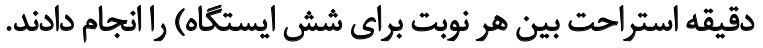

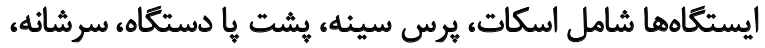

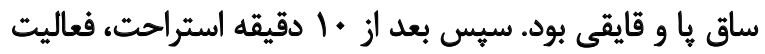

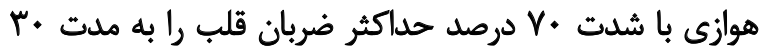

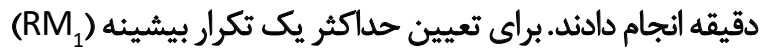

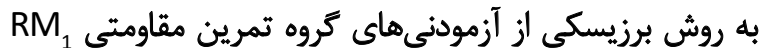

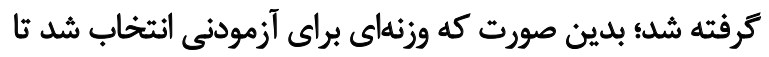

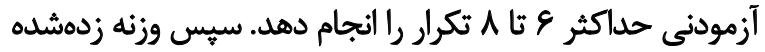

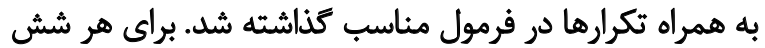

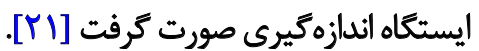

مصرف كاقئين

كروه كافئين روزانه \& ميلى كرم به ازاي هر كيلوكرم وزن

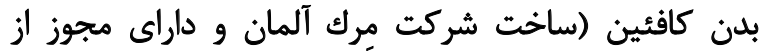

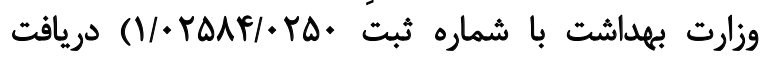

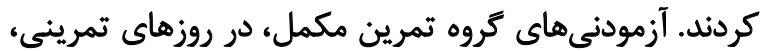

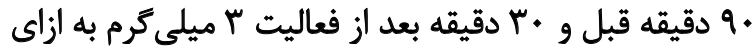

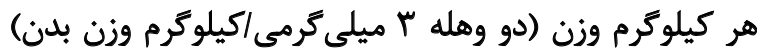

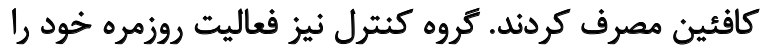

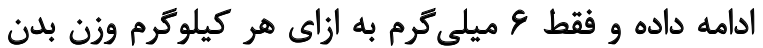

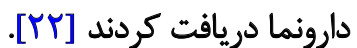

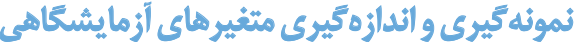

براى ارزيابى متغيرهاى بيوشيميايى، عمل خون تيرى يس إز از

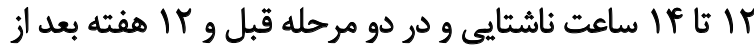

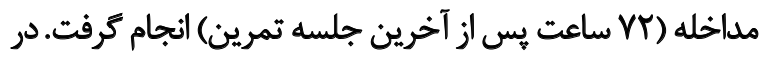

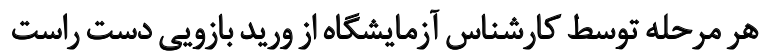

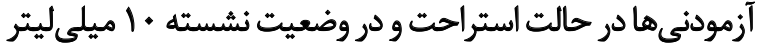

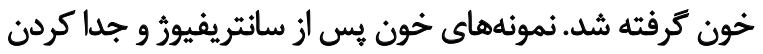

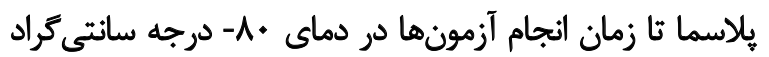


جدول ا. ميانكين و انحرافمعيار ويرّكىهاى آنترويومتريك آزمودنى ها در كروهايى مختلف

\begin{tabular}{|c|c|c|c|c|}
\hline \multirow[b]{2}{*}{ تمرين تركيبي كافئين } & \multicolumn{2}{|c|}{ ميانكين||نحرافمعيار } & & \multirow[b]{2}{*}{ متغير } \\
\hline & تمرين تركيبي & كافئين & 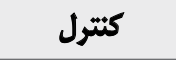 & \\
\hline$. / 4 \pm . / r q$ & $\cdot / \Psi \pm \cdot / r V$ & $r \pm \cdot / M \Lambda$ & $\cdot / \Psi \pm \cdot / v$ & سن (سال) \\
\hline$. . / 9 \pm 89 / 1 \mathrm{n}$ & $9 / 4 \pm r r / / n^{e}$ & $9) / \& \pm \wedge V / 19 q$ & SI/AITY/IV. & قد (سانتىمتر) \\
\hline$r e / q \pm r / q \Delta$ & $r / / \cdot \pm r s / q Y$ & $\Delta / / q \pm 1 . / Q$ & $r \cdot(A \pm q) / q$ & وزن (كيلوكرم) \\
\hline$V \in / \Psi \pm \Delta T / M r$ & r & PNTESNMT & $\Delta f / r \pm q 1 / r r$ & نمايه توده بدن (كيلوكرم بر متر مربع) \\
\hline
\end{tabular}

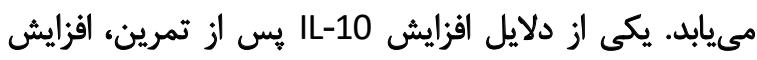

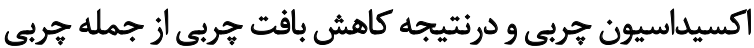

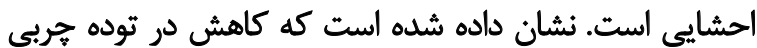

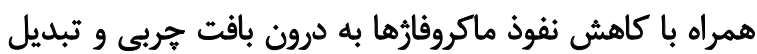

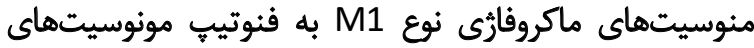

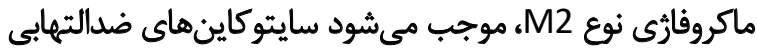

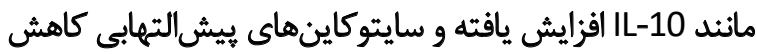

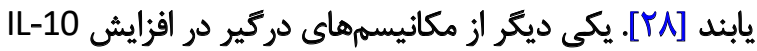

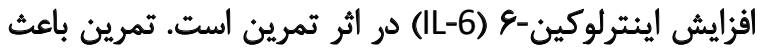

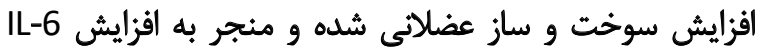

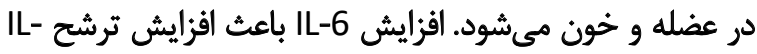

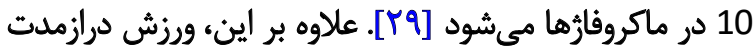

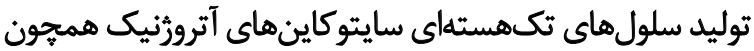

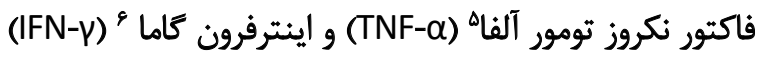

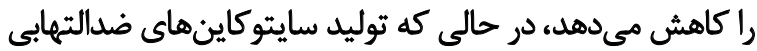

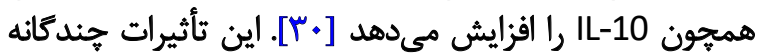

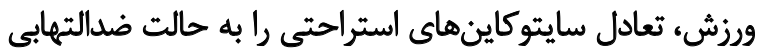

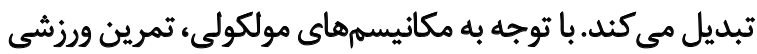

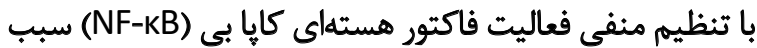

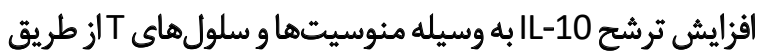

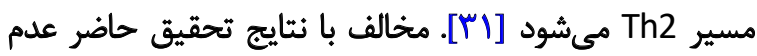

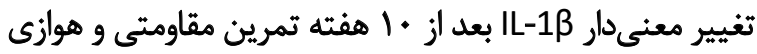

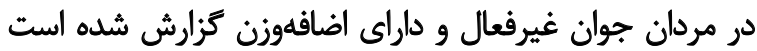

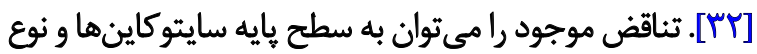

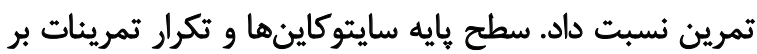

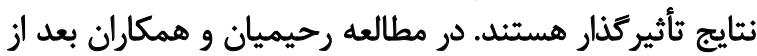

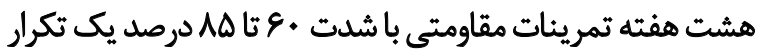

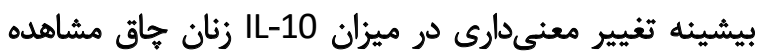

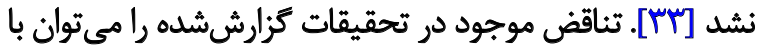

5. Tumor Necrosis Factor alpha

6. Interferon gamma
در كروه تمرين تركيبى و تمرين تركيبى - كافئين در مقايسه

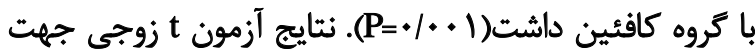

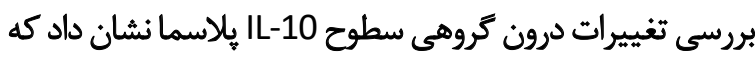

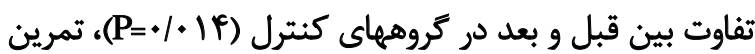

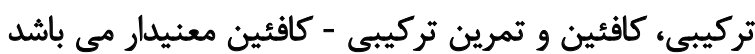

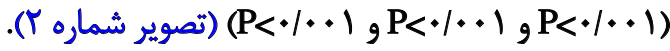

بحث

نتايج يثروهش حاضر نشان داد Y ا هفته مداخله تمرين تركيبى

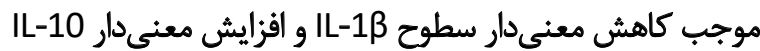

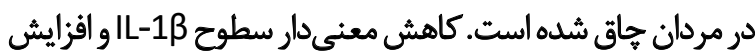

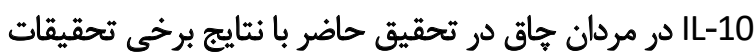

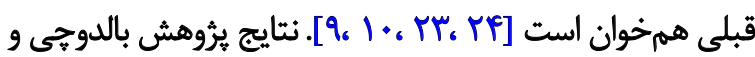

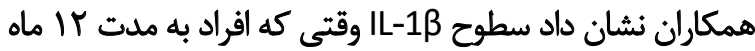

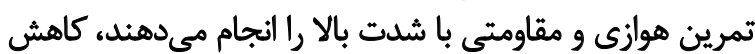

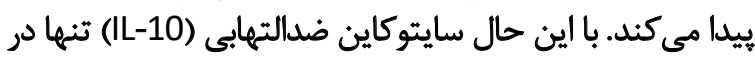

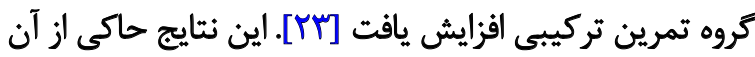

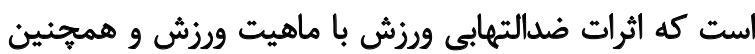

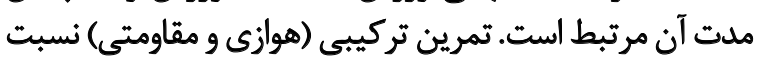

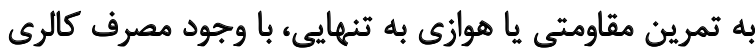

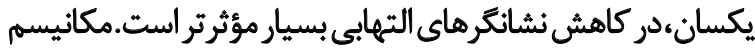

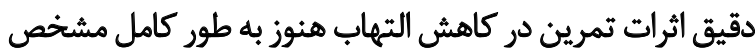

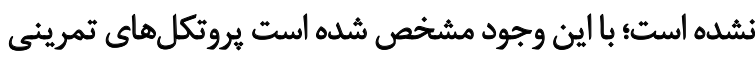

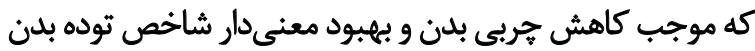

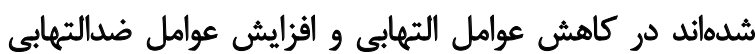

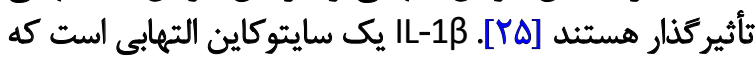

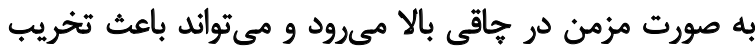

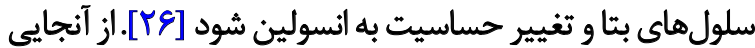

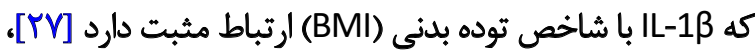

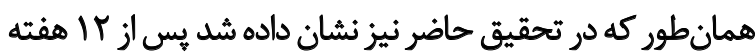

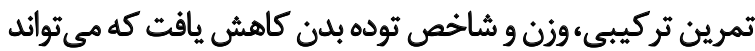

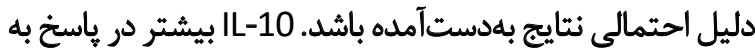

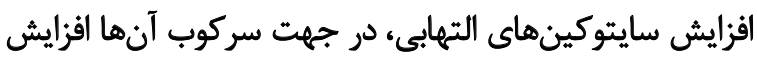




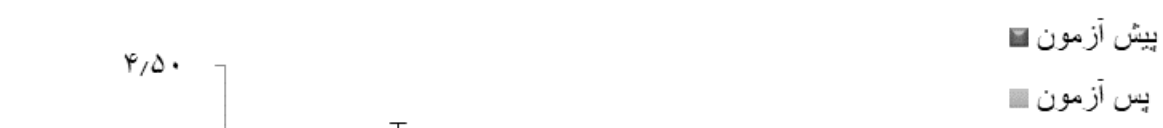

إنجان

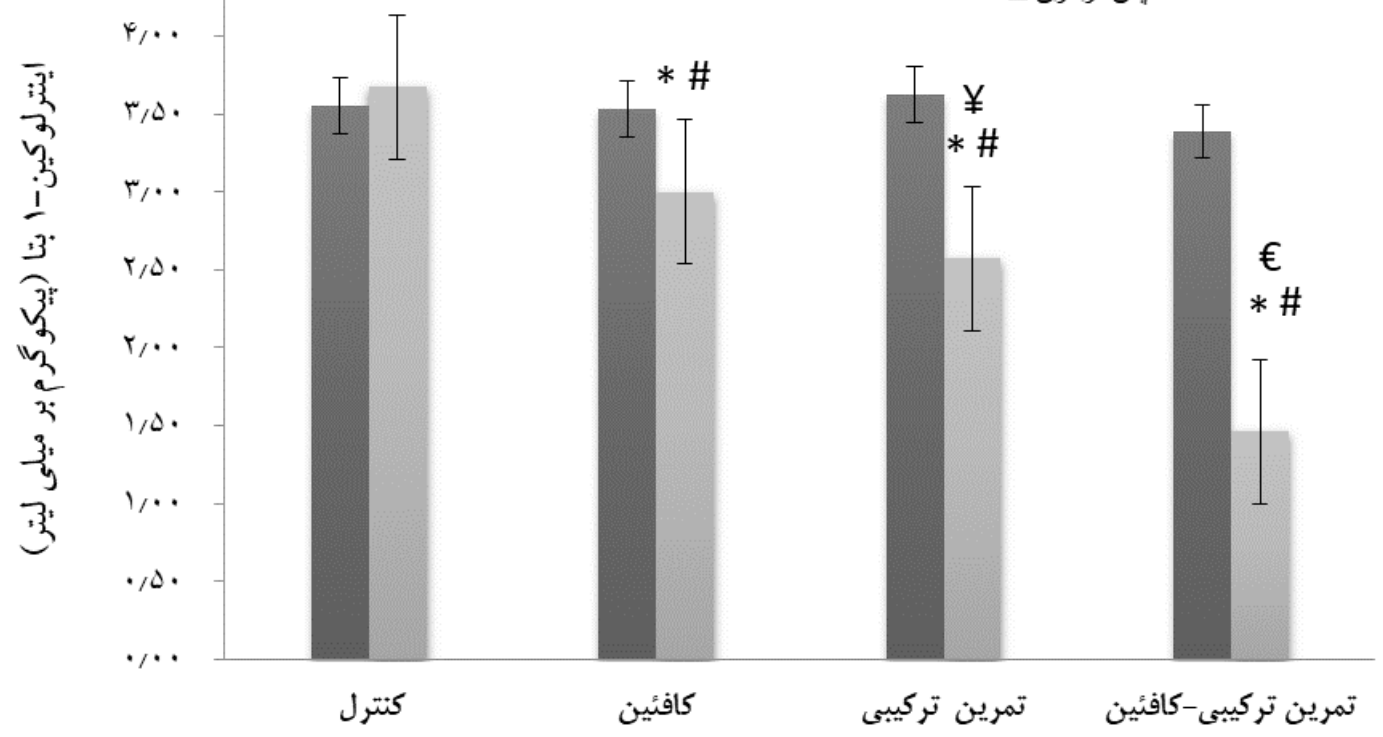

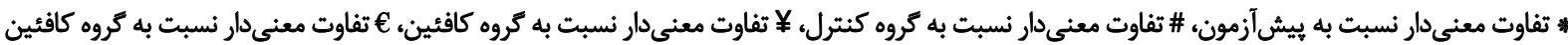
و تمرين تركيبى (AS (PS)

IL-10 در مردان جاق شده است. مطالعات كمى نشان دادهاند

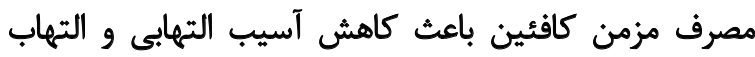

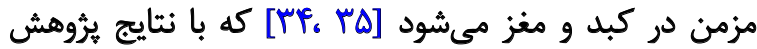

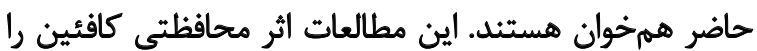

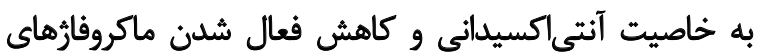
مقيم (سلولهاى كوير) و ميكروكليا نسبت دادهاند. علاوه بر اين،
عواملى از جمله وضعيت تندرستى و وزن اوليه آزمودنى ها و وطول

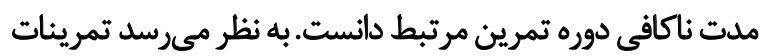

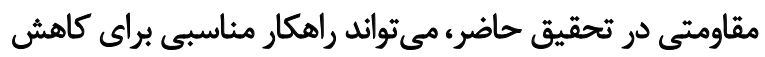

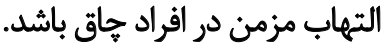

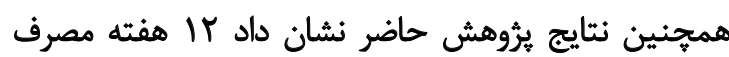

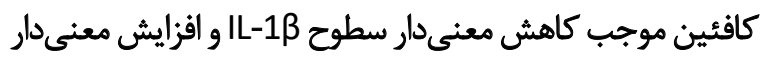

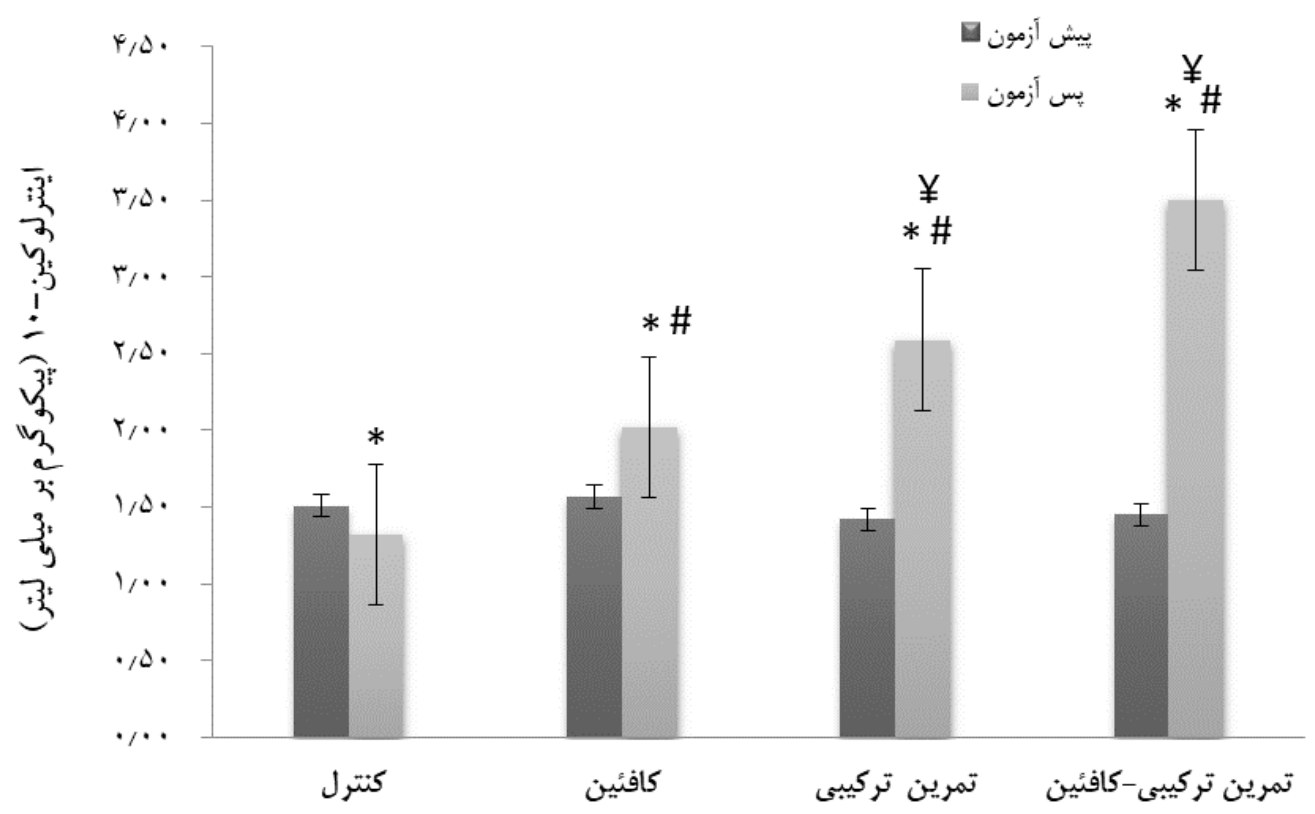

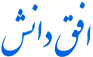

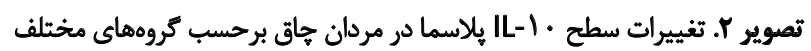

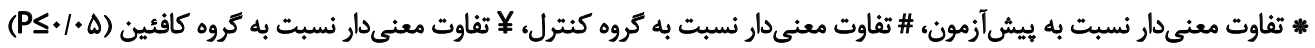


تمرين تركيبى و كافئين به عنوان يك روش درمانى جهت كاهش التهاب مزمن در جاقى مورد توجه قرافين بران ئيرد.

انجام تمرين تركيبى از نقاط قوت تحقيق حاضر بود؛ جرا كه

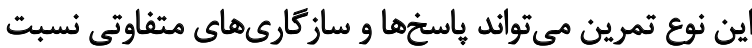

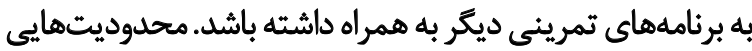

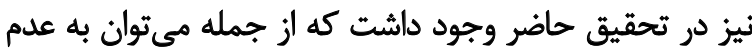

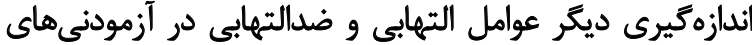

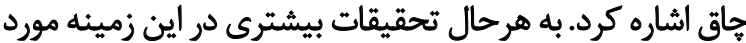

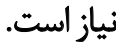

مالاحظات اخلاقي

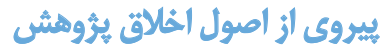

اين تحقيق با تأييد كميته اخلاق با شماره

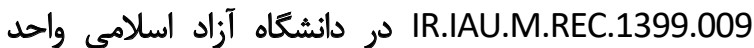

آيتالله آملى انجام شد.

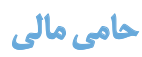

اين تحقيق مستخرج از رساله دكترى نويسنده اول در كروه

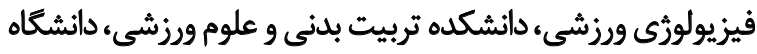

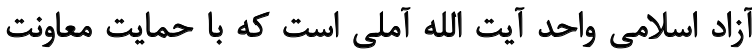

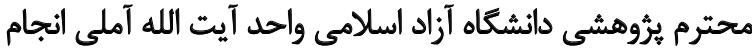

شيله أسيث.

مشاركت نويسندكان

تمام نويسندكان در آمادهازى اين مقاله مشاركت يكسان

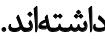

تقارض مثاقع

طبق نظر نويسندكان، هيجّونه تعارض منافعى در اين مقاله

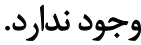

مصرف كافئين بيان سايتوكاينهاى التهابى در مونوسيتهاى

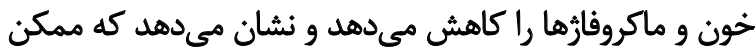

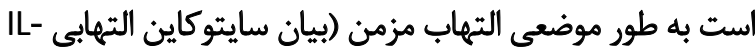

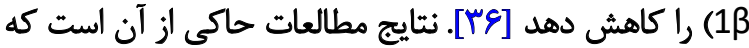

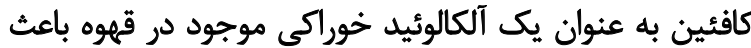

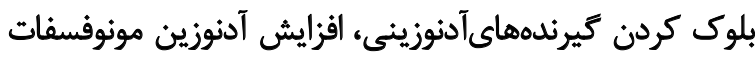

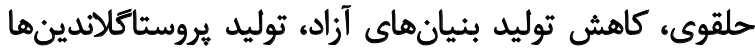

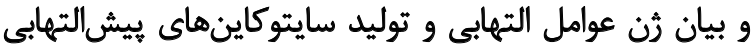

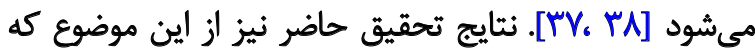

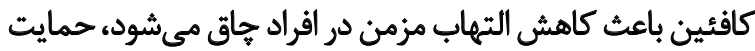

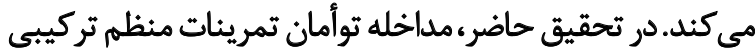

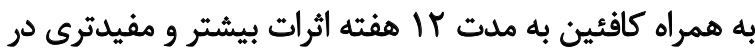

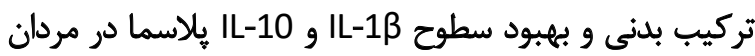

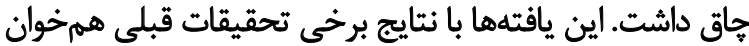

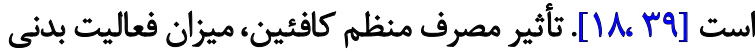

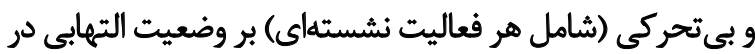

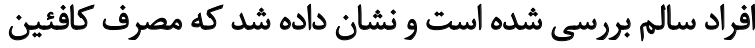

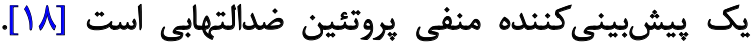

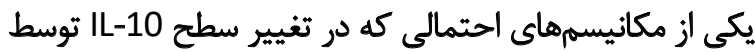

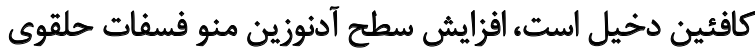

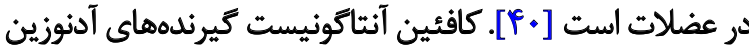

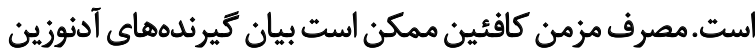

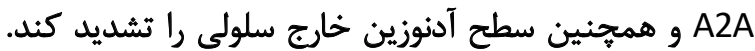

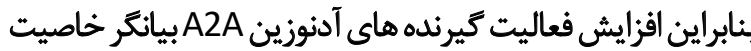

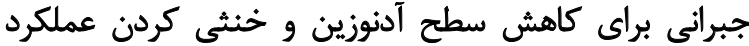

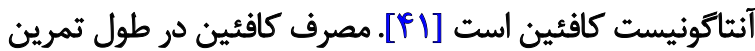

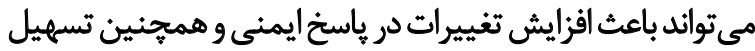

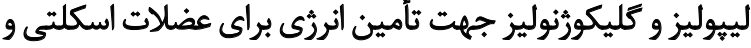

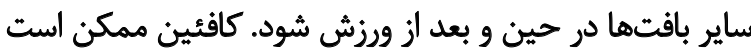

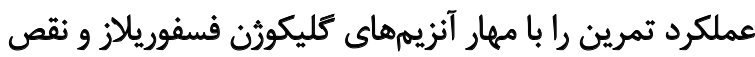

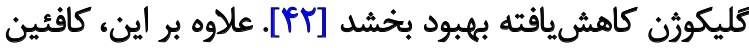

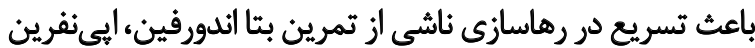

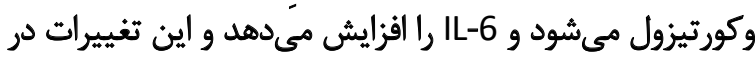

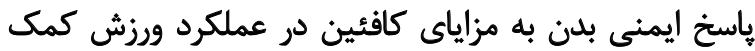

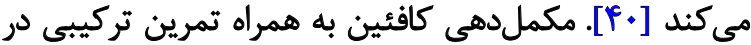

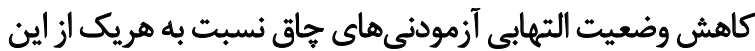

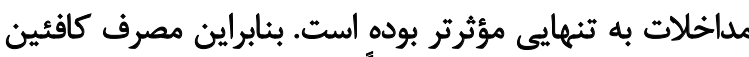

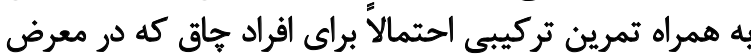

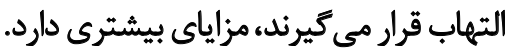

\section{تئيجليرى}

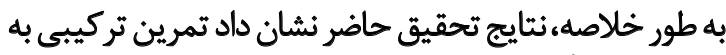

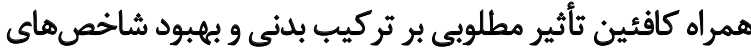

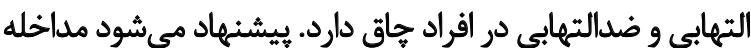




\section{References}

[1] Chooi YC, Ding C, Magkos F. The epidemiology of obesity. Metabolism. 2019; 92:6-10. [DOI:10.1016/j.metabol.2018.09.005] [PMID]

[2] Colberg SR, Sigal RJ, Fernhall B, Regensteiner JG, Blissmer BJ, Rubin RR, et al. Exercise and type 2 diabetes: the American College of Sports Medicine and the American Diabetes Association: Joint position statement. Diabetes Care. 2010; 33(12):e147-67. [DOI:10.2337/dc10-9990] [PMID] [PMCID]

[3] Moschen AR, Molnar C, Enrich B, Geiger S, Ebenbichler CF, Tilg H. Adipose and liver expression of interleukin (IL)-1 family members in morbid obesity and effects of weight loss. Molecular Medicine. 2011; 17(78):840-5. [DOI:10.2119/molmed.2010.00108] [PMID] [PMCID]

[4] Tack CJ, Stienstra R, Joosten LAB, Netea MG. Inflammation links excess fat to insulin resistance: The role of the interleukin-1 family. Immunological Reviews. 2012; 249(1):239-52. [DOI:10.1111/j.1600065X.2012.01145.x] [PMID]

[5] Zaki M, Kamal S, El-Bassyouni H, Hussein MAEA, Tawfeek HM, Girgiss $\mathrm{M}$, et al. Osteopontin, malondialdehyde and interleukin- $1 \beta$ levels in patients with insulin resistance and dyslipidemia in obese Egyptian women. Biomedical and Pharmacology Journal. 2020; 13(2):687-95. [DOI:10.13005/bpj/1922]

[6] Rahim SS, Khan N, Boddupalli CS, Hasnain SE, Mukhopadhyay S. Interleukin-10 (IL-10) mediated suppression of IL-12 production in RAW 264.7 cells also involves c-rel transcription factor. Immunology. 2005; 114(3):313-21. [DOI:10.1111/j.1365-2567.2005.02107.x] [PMID] [PMCID]

[7] Bosutti A, Malaponte G, Zanetti M, Castellino P, Heer M, Guarnieri $G$, et al. Calorie restriction modulates inactivity-induced changes in the inflammatory markers C-reactive protein and pentraxin-3. The Journal of Clinical Endocrinology and Metabolism. 2008; 93(8):32269. [DOI:10.1210/jc.2007-1684] [PMID]

[8] Chang J, Chang C, Chien EY. Association between interleukin $1 \beta$ and interleukin 10 concentrations: A cross-sectional study in young adolescents in Taiwan. BMC Pediatrics. 2013; 13:123. [DOI:10.1186/14712431-13-123] [PMID] [PMCID]

[9] Behboudi L, Eizadi M. The modifying impact of long-term endurance training on inflammatory cytokine il-1b level and vo2max in premenopausal women with abdominal obesity. Jundishapur Journal of Chronic Disease Care. 2017; 6(4):e57180. [DOl:10.5812/jjcdc.57180]

[10] Kalhor F, Arshadi S, Zafari A. [The effect the period of a resistance training on Atrogin, Eotaxin and IL-10 indices in obese women (Persian)]. Razi Journal of Medical Sciences. 2020; 27(3):130-7. http://rjms.iums.ac.ir/ article-1-6125-en.html

[11] Fatollahian Z, Monazzami A, Tadibi V, Mostafaei A. [Modulation of interleukin-1B (IL-1B), Tumor Necrosis Factor-A (TNF-A) and interleukin-10 (IL-10) genes expression following concurrent training in women with type2 diabetes (Persian)]. Iranian Journal of Diabetes and Metabolism. 2020; 19(3):160-9. http://ijdld.tums.ac.ir/article-1-5934-en.html

[12] Mohamadzadeh Salamat K, Bakhtiari N. The effects of endurance and resistance training on systemic inflammatory markers and metabolic syndrome parameters in overweight and obese men. Report of Health Care Journal. 2017; 3(3):15-26. http://jrhc.miau.ac.ir/article_2856.html

[13] Tarka SM, Hurst WJ. Introduction to the chemistry, isolation, and biosynthesis of methylxanthines. In: Spiller GA, editor. Caffeine. Boca Raton: CRC Press; 1998. p. 1-12. [DOI:10.1201/9780429126789-1]

[14] Zulak KG, Liscome DK, Ashihara H, Facchini PJ. Alkaloids. In: Crozier A, Clifford MN, Ashihara H, editors. Plant Secondary Metabolites: Occur- rence Structure, and Role in the Human Diet. Oxford: Blackwell; 2006. p.102-36. [DOI:10.1002/9780470988558.ch4]

[15] Cappelletti S, Piacentino D, Sani G, Aromatario M. Caffeine: Cognitive and physical performance enhancer or psychoactive drug? Current Neuropharmacology. 2015; 13(1):71-88. [DOI:10.2174/157015 9X13666141210215655] [PMID] [PMCID]

[16] Barcelos RP, Lima FD, Carvalho NR, Bresciani G, Royes LF. Caffeine effects on systemic metabolism, oxidative-inflammatory pathways, and exercise performance. Nutrition Research. 2020; 80:1-17 [DOI:10.1016/j.nutres.2020.05.005] [PMID]

[17] Furman D, Chang J, Lartigue L, Bolen CR, Haddad F, Gaudilliere B, et al. Expression of specific inflammasome gene modules stratifies older individuals into two extreme clinical and immunological states. Nature Medicine. 2017; 23(2):174-84 [DOI:10.1038/nm.4267] [PMID] [PMCID]

[18] Rodas L, Martinez S, Aguilo A, Tauler P. Caffeine supplementation induces higher IL-6 6 and IL-10 plasma levels in response to a treadmill exercise test. Journal of the International Society of Sports Nutrition. 2020 17(1):47. [DOI:10.1186/s12970-020-00375-4] [PMID] [PMCID]

[19] Ogunbode AM, Owolabi MO, Olayinka Ogunbode Ol, Ogunniyi A. Factors associated with obesity and weight reduction among people with obesity: A systematic review. Journal of Molecular Pathophysiology. 2020; 9(1):1-13. [DOI:10.5455/jmp.20190717044204]

[20] Jones TW, Howatson G, Russell M, French DN. Performance and endocrine responses to differing ratios of concurrent strength and endurance training. Journal of Strength and Conditioning Research. 2016; 30(3):693-702. [DOI:10.1519/JSC.0000000000001135] [PMID]

[21] Ebrahim K, Bassami M, Kolahdozi S, Karimnia Saheb V. [The effects of circuit resistance exercise on fat and carbohydrate metabolism during endurance exercise in overweight men (Persian)]. Iranian Journal of Endocrinology and Metabolism. 2012; 14(3):257-66. http://ijem.sbmu. ac.ir/article-1-1302-en.html

[22] Schubert MM, Hall S, Leveritt M, Grant G, Sabapathy S, Desbrow B. Caffeine consumption around an exercise bout: Effects on energy expenditure, energy intake, and exercise enjoyment. Journal of Applied Physiology. 2014; 117(7):745-54. [DOI:10.1152/japplphysiol.00570.2014] [PMID]

[23] Balducci S, Zanuso S, Nicolucci A, Fernando F, Cavallo S, Cardelli P, et al. Anti-inflammatory effect of exercise training in subjects with type 2 diabetes and the metabolic syndrome is dependent on exercise modalities and independent of weight loss. Nutrition, Metabolism, and Cardiovascular Diseases. 2010; 20(8):608-17. [DOI:10.1016/j.numecd.2009.04.015] [PMID]

[24] Mehrabani J, Damirchi A, Rahmaninia F. [The effect of two aerobic training intensities on lipocalin-2, interleukin-2 levels and insulin resistance index in sedentary obese men (Persian)]. Sport Physiology. 2014; 6(21):95-108. https://spj.ssrc.ac.ir/article_205.html?lang=en

[25] Selvin E, Paynter NP, Erlinger TP. The effect of weight loss on C reactive protein: A systematic review. Archives of Internal Medicine. 2007; 167(1):31-9. [DOI:10.1001/archinte.167.1.31] [PMID]

[26] Zhao G, Dharmadhikari G, Maedler K, Meyer-Hermann M. Possible role of Interleukin- $1 \beta$ in type 2 diabetes onset and implications for antiinflammatory therapy strategies. PLoS Computational Biology. 2014 10(8):e1003798. [DOI:10.1371/journal.pcbi.1003798] [PMID] [PMCID]

[27] Fève B, Bastard JP. The role of interleukins in insulin resistance and type 2 diabetes mellitus. Nature Reviews. 2009; 5(6):305-11. [DOI:10.1038/ nrendo.2009.62] [PMID] 
[28] Trayhurn P, Wood IS. Signaling role of adipose tissue: Adipokines and inflammation in obesity. Biochemical Society Transactions. 2005; 33(Pt 5):1078-81. [DOI:10.1042/BST20051078] [PMID]

[29] Lankster GI. Exercise and cytokines. Gleeson M. Immune function in sport and exercise. $2^{\text {th }}$ ed. Tehran: Hatmi 2015; 304-323.

[30] Taddei S, Galetta F, Virdis A, Ghiadoni L, Salvetti G, Franzoni F, et al. Physical activity prevents age-related impairment in nitric oxide availability in elderly athletes. Circulation. 2000; 101(25):2896-901. [DOI:10.1161/01.CIR.101.25.2896] [PMID]

[31] Nicklas BJ, Hsu FC, Brinkley TJ, Church T, Goodpaster BH, Kritchevsky $\mathrm{SB}$, et al. Exercise training and plasma c-reactive protein and interleukin-6 in elderly people. Journal of the American Geriatrics Society. 2008; 56(11):2045-52. [DOI:10.1111/j.1532-5415.2008.01994.x] [PMID] [PMCID]

[32] Taheri Kalani A, Nikseresht M. [The effect of 10 weeks resistance and aerobic training on inflammatory cytokines in sedentary overweight men (Persian)]. Journal of Iran University of Medical Science. 2015; 23(5):17-26. http://sjimu.medilam.ac.ir/article-1-2134-en.html

[33] Rahimian Mashhad Z, Attarzadeh Hosseini SR, Rashid Lamir A, Sardar MA, Nekooei S, Giti R. [Effect of aerobic and resistance exercise programs on arterial stiffness, serum IL6 and IL10 in obese women (Persian)]. The Iranian Journal of Obstetrics, Gynecology and Infertility. 2020; 23(2):20-9. http://ijogi.mums.ac.ir/article_15960.html

[34] Chen GQ, Chen YY, Wang XS, Wu SZ, Yang HM, Xu HQ, et al. Chronic caffeine treatment attenuates experimental autoimmune encephalomyelitis induced by guinea pig spinal cord homogenates in Wistar rats. Brain Research. 2010; 1309:116-25. [DOI:10.1016/j.brainres.2009.10.054] [PMID]

[35] Lv X, Chen Z, Li J, Zhang L, Liu H, Huang C, et al. Caffeine protects against alcoholic liver injury by attenuating inflammatory response and oxidative stress. Inflammation Research. 2010; 59(8):635-45. [DOI:10.1007/ s00011-010-0176-6] [PMID]

[36] Chavez-Valdez R, Wills-Karp M, Ahlawat R, Cristofalo EA, Nathan A, Gauda EB. Caffeine modulates TNF-alpha production by cord blood monocytes: The role of adenosine receptors. Pediatric Research. 2009; 65(2):203-8. [DOI:10.1203/PDR.0b013e31818d66b1] [PMID]

[37] Dray C, Daviaud D, Guigne C, Valet P, Castan-Laurell I. Caffeine reduces TNF alpha up-regulation in human adipose tissue primary culture. Journal of Physiology and Biochemistry. 2007; 63(4):329-36. [DOI:10.1007/ BF03165764] [PMID]

[38] Lee C. Antioxidant ability of caffeine and its metabolites based on the study of oxygen radical absorbing capacity and inhibition of LDL peroxidation. Clinica Chimica Acta. 2000; 295(1-2):141-54. [DOI:10.1016/ S0009-8981(00)00201-1] [PMID]

[39] Tauler P, Martinez, S, Martinez P, Lozano, L, Moreno C, Aguilo, A. Effects of caffeine supplementation on plasma and blood mononuclear cell interleukin-10 levels after exercise. International Journal of Sport Nutrition and Exercise Metabolism. 2016; 26(1):8-16. [DOI:10.1123/ ijsnem.2015-0052] [PMID]

[40] Tauler P, Martínez S, Moreno C, Monjo M, Martínez P, Aguiló A. Effects of caffeine on the inflammatory response induced by a $15-\mathrm{km}$ run competition. Medicine and Science in Sports and Exercise. 2013; 45(7):126976. [DOI:10.1249/MSS.0b013e3182857c8a] [PMID]

[41] Ribeiro JA, Sebastiao AM. Caffeine and adenosine. Journal of Alzheimer's Disease. 2010; 20(Suppl 1):S3-15. [DOI:10.3233/JAD-2010-1379] [PMID]
[42] Magkos F, Kavouras SA. Caffeine use in sports, pharmacokinetics in man, and cellular mechanisms of action. Critical Reviews in Food Science and Nutrition. 2005; 45(7-8):535-62. [DOI:10.1080/1040830491379245] [PMID] 
This Page Intentionally Left Blank 